J. Korean Math. Soc. 49 (2012), No. 3, pp. 475-491

http://dx.doi.org/10.4134/JKMS.2012.49.3.475

\title{
ALL GENERALIZED PETERSEN GRAPHS ARE UNIT-DISTANCE GRAPHS
}

\author{
Arjana Žitnik, Boris Horvat, And Tomaž Pisanski
}

\begin{abstract}
In 1950 a class of generalized Petersen graphs was introduced by Coxeter and around 1970 popularized by Frucht, Graver and Watkins. The family of $I$-graphs mentioned in 1988 by Bouwer et al. represents a slight further albeit important generalization of the renowned Petersen graph. We show that each $I$-graph $I(n, j, k)$ admits a unit-distance representation in the Euclidean plane. This implies that each generalized Petersen graph admits a unit-distance representation in the Euclidean plane. In particular, we show that every $I$-graph $I(n, j, k)$ has an isomorphic $I$-graph that admits a unit-distance representation in the Euclidean plane with a $n$-fold rotational symmetry, with the exception of the families $I(n, j, j)$ and $I(12 m, m, 5 m), m \geq 1$. We also provide unit-distance representations for these graphs.
\end{abstract}

\section{Introduction}

$I$-graphs were introduced in the Foster census [5] and form a natural generalization of the generalized Petersen graphs introduced by Coxeter [8] and named by Watkins [26]. This well-known family of graphs has been extensively studied $[1,10,18,20,22,25]$.

Let $n \geq 3$ and $j, k$ be such that $1 \leq j, k<n$ and $j, k \neq n / 2$. The $I$ graph $I(n, j, k)$ is a graph with vertex set

$$
V(I(n, j, k))=\left\{u_{0}, u_{1}, \ldots, u_{n-1}, v_{0}, v_{1}, \ldots, v_{n-1}\right\}
$$

and edge set

$$
E(I(n, j, k))=\left\{u_{i} u_{i+j}, u_{i} v_{i}, v_{i} v_{i+k} ; i=0, \ldots, n-1\right\},
$$

where the subscripts are to be read modulo $n$. Clearly, the $I$-graph $I(n, 1, k)$ is a generalized Petersen graph; we denote it also by $G(n, k)$.

Received May 14, 2010.

2010 Mathematics Subject Classification. 52C10, 05C10, 05C62, 11A99, 11Z05, 51A20, 52C30, 68R10, 05E18.

Key words and phrases. unit-distance graph, $I$-graph, generalized Petersen graph, graph representation, degenerate representation, graph isomorphism. 
In this paper we are interested in a special kind of drawings of graphs where all edges have the same length. Such a drawing of a graph $G$ is called a unitdistance representation of $G$; see, for instance, [7, 6, 9, 11, 13, 19, 21, 23].

Erdös, Hararay and Tutte [9] proposed a natural geometrical definition of the dimension of a graph $G$ and have shown that the well-known Petersen graph of Figure 1(a) can be drawn in the Euclidean plane in such a way, that vertices are mapped to distinct points in the plane and edges to line segments of length one. They proposed a non-degenerate unit-distance representation of the Petersen graph with rotational symmetry in the Euclidean plane that can be seen in Figure 1(b). Their representation respects a rotational symmetry of the Petersen graph, and their drawing is obtained from the standard drawing of the Petersen graph by suitably scaling the inner pentagram and rotating it against the outer pentagon, in such a way that the edges connecting the pentagram with the pentagon become of length one. We call this procedure $a$ twist.

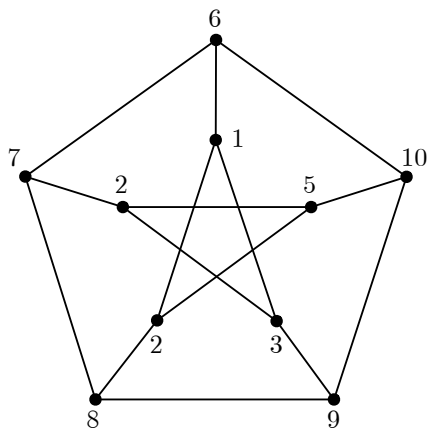

(a)

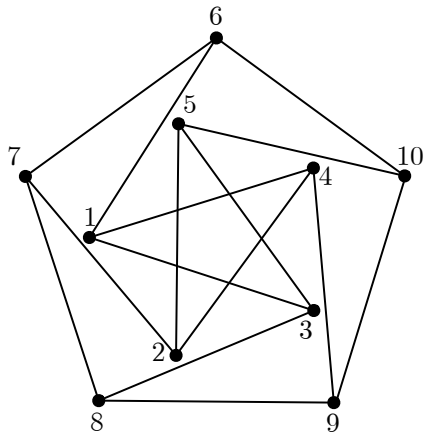

(b)

Figure 1. The well-known Petersen graph $G(5,2)$ (a) can be represented with unit distances with respect to a rotational symmetry (b).

This idea was used by S. W. Golomb [24] to draw the well-known Golomb graph, a 4-chromatic unit-distance graph with ten vertices, which can be seen in Figure 2. The twist was used again by Buckley and Harary in [6], while considering wheel graphs.

Unit-distance drawings of the Petersen graph were studied in another context, too. In [15] Horvat and Pisanski considered the vertex-degenerate unitdistance representations of the Petersen graph in the Euclidean plane; namely, drawings that respect unit edge lengths but may map two or more vertices into the same point. The vertices of the Petersen graph $G(5,2)$ in Figure 1(a) can be properly vertex-colored with three colors in such a way, that vertices 


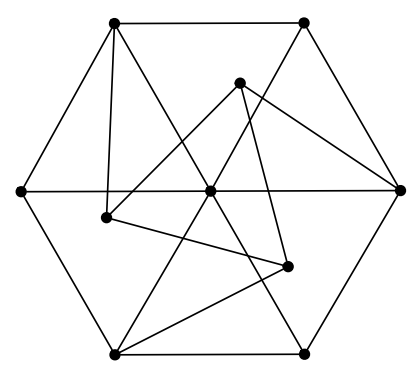

Figure 2. The Golomb graph can be realized with unitdistances using a twist on the inner triangle.

$\{1,7,9\}$ are colored with the first color, $\{4,5,6,8\}$ with the second one and vertices $\{2,3,10\}$ with the third one. This implies that the Petersen graph can be drawn in such a way that the vertices of each color class are mapped to a vertex of an equilateral triangle with side one. Since all generalized Petersen graphs are three colorable, this means that each generalized Petersen graph admits a vertex-degenerate unit-distance representation in the Euclidean plane.

In this paper we study non-vertex-degenerate unit-distance representations in the plane of the generalized Petersen graphs. If we apply a twist to obtain unit-distance representations of a generalized Petersen graph, we do not get very far. Namely, only 13 of them, including the Petersen graph, can be drawn in this way. Figure 3 depicts the other 12 generalized Petersen graphs, that have a unit-distance realization in the Euclidean plane obtained by twist: $G(6,2)$, $G(7,2), G(7,3), G(8,2), G(8,3), G(9,2), G(9,3), G(9,4), G(10,2), G(10,3)$, $G(11,2)$ and $G(12,2)$. The graph $G(10,4)$ on Figure 4 , left, is not one of the 13. However, it is isomorphic to the $I$-graph $I(10,2,3)$ that admits a unit-distance representation with rotational symmetry, see Figure 4, right. It turns out that there are many such cases. For this reason we study the unitdistance representations of generalized Petersen graphs in the broader context of $I$-graphs.

In this paper we show that for most $I$-graphs we have either a unit-distance representation obtained by using the twist or an isomorph that can be drawn in such a way. The only other connected examples are $I(12,1,5)$ and the prisms. We provide a unit-distance representation for $I(12,1,5)$ which does not use the twist while unit-distance representations for prisms were found in [16]. In the case of disconnected $I$-graphs that do not have rotational unit-distance representation we draw each copy separately. Thus, the main result of the paper is the following theorem.

Theorem 1. Each I-graph has a non-vertex degenerate unit-distance representation in the plane. 

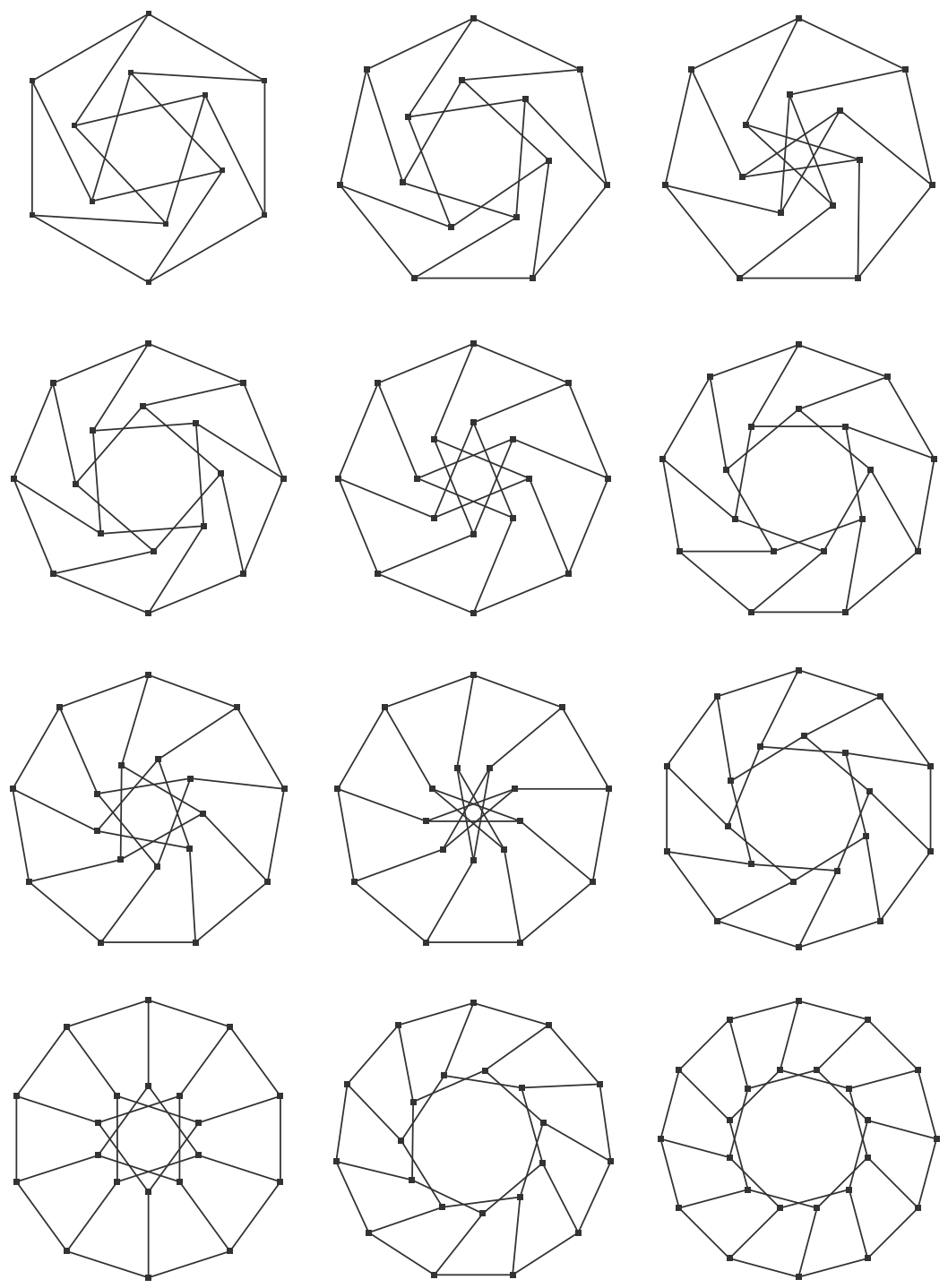

Figure 3. Apart from the Petersen graph $G(5,2)$, only twelve generalized Petersen graphs can be drawn as unit-distance graphs in the plane, using the twist.

The most of the paper is devoted to the proof of Theorem 1. We need three important tools for achieving this end:

- unit-distance representations with rotational symmetry of $I$-graphs; 


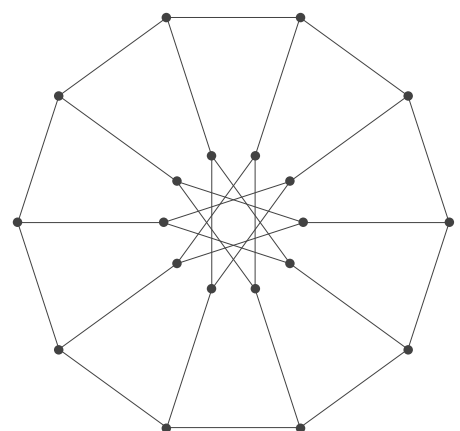

(a)

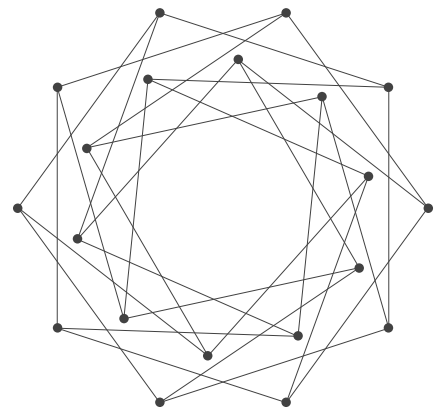

(b)

Figure 4. The generalized Petersen graph $G(10,4)$ (a) and $I$-graph $I(10,2,3)(\mathrm{b})$ are isomorphic. The former cannot be realized with unit-distances using the twist, while the second one can.

- existence of admissible isomorphs of an $I$-graph;

- finding unit-distance representations for sporadic cases.

\section{Unit-distance representations with rotational symmetry of I-graphs}

Let $G$ be a graph and let $\rho: V(G) \rightarrow \mathbb{R}^{2}$ be a representation of vertices of $G$ in the Euclidean plane such that every edge of $G$ is represented as a line segment between its end vertices. The representations of two edges may cross in the interior point. If two or more vertices of $G$ are mapped into a single point in $\mathbb{R}^{2}$, i.e., the representation $\rho$ is not injective on vertices, the representation is called vertex-degenerate. If there is an edge $e=u v$ and a vertex $w \neq u, v$ of $G$ such that $\rho(w)$ belongs to the line segment with endpoints $\rho(u)$ and $\rho(v)$, we call $\rho$ vertex-edge-degenerate. A representation is called a unit-distance representation, if $u v \in E(G)$ implies that the distance between $\rho(u)$ and $\rho(v)$ in $\mathbb{R}^{2}$ is one. In addition, we require that the representation is non-vertex-degenerate.

We focus our attention to $I$-graphs admitting non-vertex-degenerate representations with rotational symmetry. In particular, for an $I$-graph $I(n, j, k)$ we place the vertices equidistantly on two concentric rims such that vertices $u_{i}$ are placed on one rim and vertices $v_{i}$ on the other one, see Figure 5. Let $R$ be the radius of the first rim and let $r$ be the radius of the second rim. Let $\phi$ be the offset angle of the second rim with respect to the (fixed) first rim; namely, $\phi:=\angle \rho\left(u_{0}\right) \overrightarrow{0} \rho\left(v_{0}\right)$, where the point $\overrightarrow{0}$ is the center of both rims and the second rim is rotated in the counterclockwise direction according to the fixed first rim. The three parameters $R, r$ and $\phi$ determine the representation 
uniquely up to isometries of both rims. Edges between the two rims are called spokes.

The inner angle $\angle \rho\left(u_{0}\right) \overrightarrow{0} \rho\left(u_{i}\right)$ of the regular $n$-gon is clearly equal to $2 i \pi / n$. We now define the representation with rotational symmetry $\rho$ of the $I$-graph $I(n$, $j, k)$ by fixing the coordinates:

(1) $\quad \rho\left(u_{i}\right)=(R \cos (2 i \pi / n), R \sin (2 i \pi / n)), \quad i=0,1, \ldots, n-1$,

(2) $\rho\left(v_{i}\right)=(r \cos (\phi+2 i \pi / n), r \sin (\phi+2 i \pi / n)), \quad i=0,1, \ldots, n-1$,

where, in order for such a representation to be unit-distance, we have to have:

$$
R=\frac{1}{2 \sin (j \pi / n)}, \quad r=\frac{1}{2 \sin (k \pi / n)}
$$

and

$$
0 \leq|R-r| \leq 1
$$

Since $j \neq 0$ and $j \neq n / 2$, it holds $0<\sin (j \pi / n)<1$; similar observation is true for $k$. In particular $\min \{r, R\}>1 / 2$. The offset angle $\phi$ can be easily calculated from the triangle $\rho\left(u_{0}\right) \overrightarrow{0} \rho\left(v_{0}\right)$ by the law of cosines

$$
R^{2}+r^{2}-2 R r \cos (\phi)=1 .
$$

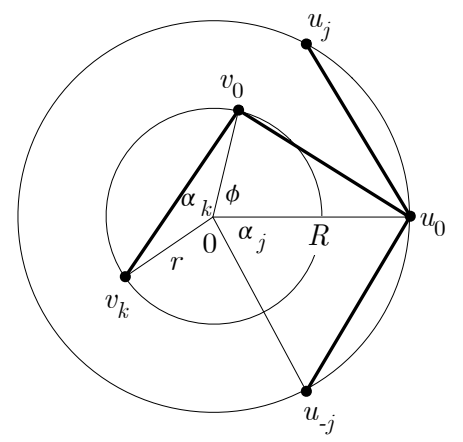

FIGURE 5. The part of the representation with the rotational symmetry with notation that will be widely used in this paper. We denote $\alpha_{j}=2 j \pi / n$ and $\alpha_{k}=2 k \pi / n$. Thick lines represent the unit-distance representations of graph edges.

Clearly, a unit-distance representation with rotational symmetry of the $I$ graph is vertex-degenerate if and only if the left inequality in (4) holds:

$$
0=|R-r| \text {. }
$$

This fact can be expressed by the parameters of the $I$-graph. 
Proposition 2. Let $\rho$ be a unit-distance representation with rotational symmetry of the $I$-graph $I(n, j, k)$. The representation $\rho$ is vertex-degenerate if and only if $j=k$.

Proof. If $j \neq k$ it follows that $R \neq r$, which, in turn, implies that the representation with rotational symmetry $\rho$ maps vertices of $I(n, j, k)$ to distinct points of $\mathbb{R}^{2}$ and $\rho$ is not vertex-degenerate.

If $j=k$, then $R=r$ and all the vertices are mapped to points on the same circle. For every point on a circle there exist at most two points on the same circle that are at distance one. Since every vertex has three neighbors, (at least) two of them must map to the same point and $\rho$ is vertex-degenerate.

Let us summarize these findings into the following theorem.

Theorem 3. An graph $I(n, j, k)$ admits a unit-distance representation with rotational symmetry if and only if

$$
0 \leq\left|\frac{1}{2 \sin (j \pi / n)}-\frac{1}{2 \sin (k \pi / n)}\right| \leq 1
$$

Furthermore, this representation is vertex-degenerate if and only if $j=k$, or equivalently

$$
0=\left|\frac{1}{2 \sin (j \pi / n)}-\frac{1}{2 \sin (k \pi / n)}\right| .
$$

Note that (7) is obtained from (4) by applying (3) and similarly (8) is obtained from (6).

We say that an $I$-graph $I(n, j, k)$ is admissible if its parameters satisfy inequality (7) and do not satisfy equality (8), otherwise it is called inadmissible. Similarly, a triple $(n, j, k)$ is admissible if the $I$-graph $I(n, j, k)$ is admissible. Unfortunately, there are infinitely many $I$-graphs that do not meet the conditions of Theorem 3 , the smallest example being $I(10,1,4)$ alias generalized Petersen graph $G(10,4)$. In the next section, the question which $I$-graphs have admissible isomorphs will be studied.

The following result shows that we can get quite a large range of admissible I-graphs.

Lemma 4. A graph $I(n, j, k)$ admits a unit-distance representation with rotational symmetry that is not vertex-degenerate if $j, k \in\left[\frac{n}{9}, \frac{8 n}{9}\right]$.

Proof. If $j \in\left[\frac{n}{9}, \frac{8 n}{9}\right], \sin (j \pi / n) \geq \sin (\pi / 9)>0$ and since $j \neq \frac{n}{2}$, we also have $\sin (j \pi / n)<\sin (\pi / 2)=1$. The same holds for $k$. Thus

$$
\left|\frac{1}{2 \sin (j \pi / n)}-\frac{1}{2 \sin (k \pi / n)}\right| \leq \frac{1}{2 \sin (\pi / 9)}-\frac{1}{2 \sin (\pi / 2)} \leq 1
$$

and the assertion follows by Theorem 3 . 
We call a graph $I(n, j, k)$ or a triple $(n, j, k)$ strongly admissible if $j, k \in$ $\left[\frac{n}{9}, \frac{8 n}{9}\right]$. Similarly, we call a pair $(n, j)$ strongly admissible if $j \in\left[\frac{n}{9}, \frac{8 n}{9}\right]$. The smallest admissible $I$-graph that is not strongly admissible is the renowned Desargues graph $I(10,1,3)$. Thus, the condition of Lemma 4 is not a necessary condition for an $I$-graph to be admissible.

\section{Existence of admissible isomorphs of $I$-graphs}

A natural strategy for proving that an $I$-graph $I(n, j, k)$ is a unit-distance graph would be to either prove that $I(n, j, k)$ is admissible or to prove that it has an admissible isomorph. Therefore checking isomorphisms of $I$-graphs is important. The following result, recently proven in [17], see also [4], determines the collection of isomorphs of a given $I$-graph.

Theorem 5. Given two I-graphs $I(n, j, k)$ and $I\left(n, j_{1}, k_{1}\right)$, they are isomorphic if and only if there exists an integer a, relatively prime to $n$, such that either $\left\{j_{1}, k_{1}\right\}=\{a j \bmod n, a k \bmod n\}$ or $\left\{j_{1}, k_{1}\right\}=\{a j \bmod n,-a k \bmod n\}$.

The following result shows that a vast majority of $I$-graphs have strongly admissible isomorphs. Only two sporadic families remain which have to be addressed by methods that are not using the twist.

Theorem 6. Let $n, j, k$ be positive integers such that $1 \leq j<k<n / 2$ and the triple $(n, j, k)$ is not of the form $(12 m, m, 5 m)$, where $m \in \mathbb{N}$. Then there exists an integer $a \in \mathbb{Z}_{n}^{*}$ such that the triple $(n, a j, a k)$ is admissible.

Before we prove Theorem 6 we first need some technical lemmas. Lemma 10 shows that in most cases we can find strongly admissible equivalents. In its proof we use the fact from Lemma 9, namely many pairs have sufficiently many strongly admissible equivalents. Its proof relies on the following numbertheoretical lemma. By $\varphi$ we denote the Euler totient function.

Lemma 7. Let $n \geq 3$ be an integer and $n \notin\{10,12\}$. Then

$$
\left|\mathbb{Z}_{n}^{*} \cap[1,\lceil n / 9\rceil-1]\right|<\varphi(n) / 4 .
$$

Proof. Let $n=p_{1}^{k_{1}} p_{2}^{k_{2}} \cdots p_{t}^{k_{t}}$ be the prime factorization of $n$. Using the inclusion-exclusion principle, we can calculate the number of positive integers less than $n$ that are coprime to $n$ as

$$
\begin{aligned}
\varphi(n)= & n-1-\sum_{i=1}^{t}\left(\frac{n}{p_{i}}-1\right)+\sum_{i, j: 1 \leq i<j \leq t}\left(\frac{n}{p_{i} p_{j}}-1\right) \\
& -\sum_{i, j, \ell: 1 \leq i<j<\ell \leq t}\left(\frac{n}{p_{i} p_{j} p_{\ell}}-1\right)+\cdots+(-1)^{t}\left(\frac{n}{p_{1} p_{2} \cdots p_{t}}-1\right) .
\end{aligned}
$$


Denote by $x$ the number of positive integers less than $n / 9$ that are coprime to $n$. Then

$$
\begin{aligned}
x= & \left\lceil\frac{n}{9}\right\rceil-1-\sum_{i=1}^{t}\left(\left\lceil\frac{n}{9 p_{i}}\right\rceil-1\right)+\sum_{i, j: 1 \leq i<j \leq t}\left(\left\lceil\frac{n}{9 p_{i} p_{j}}\right\rceil-1\right) \\
& -\sum_{i, j, \ell: 1 \leq i<j<\ell \leq t}\left(\left\lceil\frac{n}{9 p_{i} p_{j} p_{\ell}}\right\rceil-1\right)+\cdots+(-1)^{t}\left(\left\lceil\frac{n}{9 p_{1} p_{2} \cdots p_{t}}\right\rceil-1\right) .
\end{aligned}
$$

To show that $x$ does not differ too much from $\varphi(n) / 9$, we simply subtract the above expressions. Note that for any integer $a$, the number $\lceil a / 9\rceil$ is greater than $a / 9$ by at most $8 / 9$.

$$
\begin{aligned}
\left|\frac{\varphi(n)}{9}-x\right|= & \mid \frac{n}{9}-\frac{1}{9}-\left\lceil\frac{n}{9}\right\rceil+1-\sum_{i=1}^{t}\left(\frac{n}{9 p_{i}}-\frac{1}{9}-\left\lceil\frac{n}{9 p_{i}}\right\rceil+1\right) \\
& +\sum_{i, j: 1 \leq i<j \leq t}\left(\frac{n}{9 p_{i} p_{j}}-\frac{1}{9}-\left\lceil\frac{n}{9 p_{i} p_{j}}\right\rceil+1\right) \\
& -\sum_{i, j, \ell: 1 \leq i<j<\ell \leq t}\left(\frac{n}{9 p_{i} p_{j} p_{\ell}}-\frac{1}{9}-\left\lceil\frac{n}{9 p_{i} p_{j} p_{\ell}}\right\rceil+1\right) \\
& +\cdots+(-1)^{t}\left(\frac{n}{9 p_{1} p_{2} \cdots p_{t}}-\frac{1}{9}-\left\lceil\frac{n}{9 p_{1} p_{2} \cdots p_{t}}\right]+1\right) \mid \\
\leq & \frac{8}{9}+\sum_{i=1}^{t} \frac{8}{9}+\sum_{i, j: 1 \leq i<j \leq t} \frac{8}{9}+\sum_{i, j, \ell: 1 \leq i<j<\ell \leq t}+\cdots+\frac{8}{9} \\
= & \frac{8}{9}\left(1+\left(\begin{array}{l}
t \\
1
\end{array}\right)+\left(\begin{array}{l}
t \\
2
\end{array}\right)+\cdots+\left(\begin{array}{l}
t \\
t
\end{array}\right)\right)=\frac{8}{9} 2^{t} .
\end{aligned}
$$

We now show that $x<\varphi(n) / 4$ if $n \notin\{10,12\}$. If $t=1$, then $\varphi(n) / 9-x=$ $n / 9-\lceil n / 9\rceil-n /\left(9 p_{1}\right)+\left\lceil n /\left(9 p_{1}\right)\right\rceil$ and there are three cases to consider. If $n$ is divisible by $9 p_{1}$, then $n$ is also divisible by 9 and $x=\varphi(n) / 9$. If $n$ is divisible by 9 , but it is not divisible by $9 p_{1}$, then $x=\varphi(n) / 9-1$. In the last case where $n$ is not divisible by 9 , we also have $x=\varphi(n) / 9$. In any case, $x<\varphi(n) / 4$.

If $t \geq 2$, then one can verify that $\varphi(n)>7 \cdot 2^{t}$ except when $n$ is one of the numbers from Table 1 . For any $n$ from this table it is easy verify that $x<\varphi(n) / 4$ if $n \notin\{10,12\}$. For other numbers with $t \geq 2$ we consider two cases. If $x \leq \varphi(n) / 9$, then also $x<\varphi(n) / 4$. Otherwise

and

$$
x-\frac{\varphi(n)}{9} \leq \frac{8}{9} 2^{t}<\frac{8}{9} \cdot \frac{\varphi(n)}{7}
$$

$$
x<\frac{\varphi(n)}{9}+\frac{8}{63} \varphi(n)=\frac{15}{63} \varphi(n)<\frac{\varphi(n)}{4} .
$$

This completes the proof. 
TABLE 1. Positive integers with $t$ distinct prime factors having $\varphi(n)<7 \cdot 2^{t}$.

\begin{tabular}{|l|l|l|}
\hline$n$ & $\varphi(n)$ & $x$ \\
\hline $2 \cdot 3$ & 2 & 0 \\
$\mathbf{2}^{\mathbf{2}} \cdot \mathbf{3}$ & $\mathbf{4}$ & $\mathbf{1}$ \\
$2^{3} \cdot 3$ & 8 & 1 \\
$2^{4} \cdot 3$ & 16 & 2 \\
$2 \cdot 3^{2}$ & 6 & 1 \\
$2^{2} \cdot 3^{2}$ & 12 & 1 \\
$2^{3} \cdot 3^{2}$ & 24 & 3 \\
$2 \cdot 3^{3}$ & 18 & 2 \\
$\mathbf{2} \cdot \mathbf{5}$ & $\mathbf{4}$ & $\mathbf{1}$ \\
$2^{2} \cdot 5$ & 8 & 1 \\
$2^{3} \cdot 5$ & 16 & 2 \\
$2 \cdot 5^{2}$ & 20 & 2 \\
$2 \cdot 7$ & 6 & 1 \\
$2^{2} \cdot 7$ & 12 & 2 \\
$2^{3} \cdot 7$ & 24 & 3 \\
$2 \cdot 11$ & 10 & 1 \\
$2^{2} \cdot 11$ & 20 & 2 \\
$2 \cdot 13$ & 12 & 1 \\
\hline$t=2: 7 \cdot 2^{t}=28$
\end{tabular}

\begin{tabular}{|l|l|l|}
\hline$n$ & $\varphi(n)$ & $x$ \\
\hline $2^{2} \cdot 13$ & 24 & 3 \\
$2 \cdot 17$ & 16 & 2 \\
$2 \cdot 19$ & 18 & 2 \\
$2 \cdot 23$ & 22 & 3 \\
$3 \cdot 5$ & 8 & 1 \\
$3^{2} \cdot 5$ & 24 & 3 \\
$3 \cdot 7$ & 12 & 2 \\
$3 \cdot 11$ & 20 & 2 \\
$3 \cdot 13$ & 24 & 3 \\
$5 \cdot 7$ & 24 & 3 \\
\hline $2 \cdot 3 \cdot 5$ & 8 & 1 \\
$2^{2} \cdot 3 \cdot 5$ & 16 & 1 \\
$2^{3} \cdot 3 \cdot 5$ & 32 & 4 \\
$2 \cdot 3^{2} \cdot 5$ & 24 & 2 \\
$2^{2} \cdot 3^{2} \cdot 5$ & 48 & 6 \\
$2 \cdot 3 \cdot 5^{2}$ & 40 & 4 \\
$2 \cdot 3 \cdot 7$ & 12 & 1 \\
$2^{2} \cdot 3 \cdot 7$ & 24 & 2 \\
\hline \multicolumn{2}{|l}{$t=3: 7 \cdot 2^{t}=56$}
\end{tabular}

\begin{tabular}{|l|l|l|}
\hline$n$ & $\varphi(n)$ & $x$ \\
\hline $2^{3} \cdot 3 \cdot 7$ & 48 & 5 \\
$2 \cdot 3^{2} \cdot 7$ & 36 & 4 \\
$2 \cdot 3 \cdot 11$ & 20 & 3 \\
$2^{2} \cdot 3 \cdot 11$ & 40 & 4 \\
$2 \cdot 3 \cdot 13$ & 24 & 3 \\
$2^{2} \cdot 3 \cdot 13$ & 48 & 5 \\
$2 \cdot 3 \cdot 17$ & 32 & 4 \\
$2 \cdot 3 \cdot 19$ & 36 & 4 \\
$2 \cdot 3 \cdot 23$ & 44 & 5 \\
$2 \cdot 5 \cdot 7$ & 24 & 2 \\
$2^{2} \cdot 5 \cdot 7$ & 48 & 5 \\
$2 \cdot 5 \cdot 11$ & 40 & 4 \\
$2 \cdot 5 \cdot 13$ & 48 & 5 \\
$3 \cdot 5 \cdot 7$ & 48 & 5 \\
\hline $2 \cdot 3 \cdot 5 \cdot 7$ & 48 & 6 \\
$2^{2} \cdot 3 \cdot 5 \cdot 7$ & 96 & 11 \\
$2 \cdot 3 \cdot 5 \cdot 11$ & 80 & 8 \\
$2 \cdot 3 \cdot 5 \cdot 13$ & 96 & 11 \\
\hline \multicolumn{2}{|l|}{$t=4: 7 \cdot 2^{t}=112}$.
\end{tabular}

Remark 8. Note that for $n \in\{10,12\}$ we have the equality in (9). Namely, in this case $\varphi(n) / 4=1$ and there exists exactly one element from $\mathbb{Z}_{n}^{*}$ that is smaller than $n / 9$.

Lemma 9. Let $n$ and $k$ be positive integers such that $1 \leq k<n / 2$. Let $A=\left\{a k ; a \in \mathbb{Z}_{n}^{*}\right\}$ be a multiset. If $n / \operatorname{gcd}(n, k) \in\{10,12\}$, then exactly $\varphi(n) / 4$ of the elements of $A$ belong to the interval $[1,\lceil n / 9\rceil-1]$. Otherwise strictly less than $\varphi(n) / 4$ of the elements of $A$ belong to the interval $[1,\lceil n / 9\rceil-1]$.

Proof. Denote $n_{k}=\operatorname{gcd}(n, k)$. Then $n$ and $k$ can be written as products $n=n^{\prime} n_{k}$ and $k=k^{\prime} n_{k}$ where $\operatorname{gcd}\left(n^{\prime}, k^{\prime}\right)=1$. First we calculate the multiplicity of an arbitrary element $a k$ of $A$. For this purpose we check for which numbers $b \in \mathbb{Z}_{n}$ we have $a k \equiv b k(\bmod n)$. This congruence is satisfied if and only if $a k^{\prime} \equiv b k^{\prime}\left(\bmod n^{\prime}\right)$ which is true if and only if $a \equiv b\left(\bmod n^{\prime}\right)$ since $n^{\prime}$ and $k^{\prime}$ are coprime. Then we can write $b=a+t n^{\prime}$, where $0 \leq t<n_{k}$. The number $b$ is coprime to $n$ if and only if $t$ is divisible by every prime that divides $n$ and does not divide $n^{\prime}$. There are $\varphi(n) / \varphi\left(n^{\prime}\right)$ possible choices for $t$ and thus also $\varphi(n) / \varphi\left(n^{\prime}\right)$ choices for $b$. It follows that the multiplicity of $a k$ in $A$ is $\varphi(n) / \varphi\left(n^{\prime}\right)$. Moreover, the mapping $f: a k \mapsto a k^{\prime}$ maps distinct elements of $A$ to distinct elements of $\mathbb{Z}_{n^{\prime}}^{*}$. 
Take an element $x \in \mathbb{Z}_{n^{\prime}}^{*}$. By the above comments there exists an $a \in \mathbb{Z}_{n}^{*}$ such that $x=a k^{\prime}$. Therefore $f\left(n_{k} x\right)=x$. Thus every element $x$ from $\mathbb{Z}_{n^{\prime}}^{*}$ corresponds to $\varphi(n) / \varphi\left(n^{\prime}\right)$ copies of $n_{k} x$ from $A$. Moreover, $x<n^{\prime} / 9$ if and only if $n_{k} x<n^{\prime} n_{k} / 9=n / 9$. Since

$$
\frac{\varphi\left(n^{\prime}\right)}{4} \cdot \frac{\varphi(n)}{\varphi\left(n^{\prime}\right)}=\frac{\varphi(n)}{4},
$$

exactly $\varphi(n) / 4$ of elements from $A$ are smaller than $n / 9$ if $n^{\prime} \in\{10,12\}$ and otherwise strictly less than $\varphi(n) / 4$ elements from $\mathbb{Z}_{n}^{*}$ are smaller than $n / 9$ by Lemma 7 and Remark 8.

Lemma 10. Let $n, j, k$ be positive integers such that $1 \leq j, k<n / 2$. Denote $m=\operatorname{gcd}(n, j, k), n_{j}=\operatorname{gcd}(n, j) / m, n_{k}=\operatorname{gcd}(n, k) / m$ and $n^{\prime}=n /\left(m n_{j} n_{k}\right)$. If $n^{\prime} n_{j} \notin\{10,12\}$ or $n^{\prime} n_{k} \notin\{10,12\}$, then there exists an $a \in \mathbb{Z}_{n}^{*}$ such that $a j \in[n / 9,8 n / 9]$ and $a k \in[n / 9,8 n / 9]$.

Proof. Denote by $A$ the multiset $\left\{a k ; a \in \mathbb{Z}_{n}^{*}\right\}$ and by $B$ the multiset $\{a j ; a \in$ $\left.\mathbb{Z}_{n}^{*}\right\}$. If $n^{\prime} n_{k} \notin\{10,12\}$, then strictly less than $\varphi(n) / 4$ of the elements of $A$ belong to the interval $[1,\lceil n / 9\rceil-1]$ by Lemma 9 . Since $a j=-(-a) j \equiv$ $n-(-a) j(\bmod n)$, also less than $\varphi(n) / 4$ elements of $A$ belong to the interval $[\lfloor 8 n / 9\rfloor+1, n-1]$. It follows that more than $\varphi(n) / 2$ of elements of $A$ belong to the interval $[n / 9,8 n / 9]$. If $n^{\prime} n_{k} \in\{10,12\}$, then exactly $\varphi(n) / 2$ of elements of $A$ belong to the interval $[n / 9,8 n / 9]$. Similarly, if $n^{\prime} n_{k} \notin\{10,12\}$, then more than $\varphi(n) / 2$ elements of $B$ belong to the interval $[n / 9,8 n / 9]$ and if $n^{\prime} n_{k} \in\{10,12\}$, exactly $\varphi(n) / 2$ elements of $B$ belong to the interval $[n / 9,8 n / 9]$. Thus, if not both $n^{\prime} n_{j}$ and $n^{\prime} n_{k}$ belong to $\{10,12\}$, it is not possible to have $\varphi(n)$ pairs $(a j, a k)$ such that at least one of $a j$ or $a k$ does not belong to the interval $[n / 9,8 n / 9]$. Therefore there must exist an $a \in \mathbb{Z}_{n}^{*}$ such that both $a j$ and $a k$ belong to the interval $[n / 9,8 n / 9]$.

Proof of Theorem 6. Denote $m=\operatorname{gcd}(n, j, k), n_{j}=\operatorname{gcd}(n, j) / m, n_{k}=\operatorname{gcd}(n$, $k) / m, n^{\prime}=n /\left(m n_{j} n_{k}\right), j^{\prime}=j /\left(m n_{j}\right)$ and $k^{\prime}=k /\left(m n_{k}\right)$. Note that $\operatorname{gcd}\left(n_{j}, n_{k}\right)$ $=1$ and $\operatorname{gcd}\left(n^{\prime}, j^{\prime}\right)=\operatorname{gcd}\left(n^{\prime}, k^{\prime}\right)=\operatorname{gcd}\left(j^{\prime}, n_{k}\right)=\operatorname{gcd}\left(k^{\prime}, n_{j}\right)=1$.

If $n^{\prime} n_{j} \notin\{10,12\}$ or $n^{\prime} n_{k} \notin\{10,12\}$, then there exists an $a \in \mathbb{Z}_{n}^{*}$ such that $a j \in[n / 9,8 n / 9]$ and $a k \in[n / 9,8 n / 9]$ by Lemma 10 . Following Lemma 4 , the triple $(n, a j, a k)$ is admissible.

Otherwise there are three cases to consider. If $n^{\prime} n_{j}=n^{\prime} n_{k}=10$, then $n^{\prime}=10$ and $n_{j}=n_{k}=1$ since $n_{j}$ and $n_{k}$ are coprime. Since also $j^{\prime}$ and $k^{\prime}$ are coprime to $n^{\prime}$, the only possible triples are of the form $(10 m, m, 3 m)$, which are admissible by Theorem 3 .

We use the same reasoning in the case $n^{\prime} n_{j}=n^{\prime} n_{k}=12$ to obtain possible triples of the form $(12 m, m, m)$ or $(12 m, m, 5 m)$, which we do not consider in this theorem.

The last case to consider is $n^{\prime} n_{j}, n^{\prime} n_{k} \in\{10,12\}$ and $n^{\prime} n_{j} \neq n^{\prime} n_{k}$. Then $n^{\prime}=2, n_{j}=5$ and $n_{k}=6$ or $n^{\prime}=2, n_{j}=6$ and $n_{k}=5$. Since $j^{\prime}$ and $k^{\prime}$ are both 
coprime to $n^{\prime}, \operatorname{gcd}\left(j^{\prime}, n_{k}\right)=\operatorname{gcd}\left(k^{\prime}, n_{j}\right)=1$ and $1 \leq j<k<n / 2$, we obtain possible triples of the form $(60 m, 5 m, 6 m),(60 m, 5 m, 18 m),(60 m, 6 m, 25 m)$ and $(60 m, 18 m, 25 m)$. For any $m \geq 1$, the triple $(60 m, 5 m, 6 m)$ is admissible by Theorem 3 . Taking $a=11, a=41$ and $a=7$, respectively, the triples $(60 m, a \cdot 5 m, a \cdot 18 m),(60 m, a \cdot 6 m, a \cdot 25 m)$ and $(60 m, a \cdot 18 m, a \cdot 25 m)$ are also admissible.

\section{Sporadic cases and proof of the main theorem}

Let us observe special non-vertex-degenerate unit-distance representations of the two families of $I$-graphs that are not covered by Theorem 6 . To deal with non-connected cases we use the following result from [4].

Proposition 11. Let $n, j, k$ be positive integers such that $1 \leq j, k<n$ and $j, k \neq n / 2$. The graph $I(n, j, k)$ is connected if and only if $\operatorname{gcd}(n, j, k)=$ 1. If $\operatorname{gcd}(n, j, k)=d>1$, then the graph $I(n, j, k)$ consists of $d$ copies of $I(n / d, j / d, k / d)$.

Proposition 12. Let $n \geq 3$ and $j$ be positive integers such that $1 \leq j<n$ and $j \neq n / 2$. The $I$-graph $I(n, j, j)$ has a non-vertex-degenerate unit-distance representation in the plane.

Proof. Let $d=\operatorname{gcd}(n, j)$. Observe, that an $I$-graph $I(n, j, j)$ is a graph union of $d(n / d)$-prisms. Each $(n / d)$-prism can be constructed as the Cartesian product of the cycle $C_{n / d}$ on $n / d$ vertices and the complete graph on two vertices $K_{2}$. Following [16, Theorem 3.4], the graph $I(n, j, j)$ has a non-vertex-degenerate unit-distance realization in $\mathbb{R}^{2}$.

Proposition 13. The I-graph $I(12,1,5)$ has a unit-distance representation in the plane that is not vertex-degenerate.

Proof. We construct a unit-distance representation $\rho$ of $I(12,1,5)$ by placing its vertices on four concentric cycles as follows:

$$
\begin{aligned}
\rho\left(u_{i}\right) & =(R \cos (i \pi / 6), R \sin (i \pi / 6)), \quad i=0,2,4,6,8,10, \\
\rho\left(u_{i}\right) & =(r \cos (i \pi / 6), r \sin (i \pi / 6)), \quad i=1,3,5,7,9,11, \\
\rho\left(v_{i}\right) & =(L \cos (i \pi / 6), L \sin (i \pi / 6)), \quad i=0,2,4,6,8,10, \\
\rho\left(v_{i}\right) & =(\ell \cos (i \pi / 6), \ell \sin (i \pi / 6)), \quad i=1,3,5,7,9,11 .
\end{aligned}
$$

In order for $\rho$ to be unit-distance representation, we have the following relationships between the radii: $L=R-1, \ell=r-1,1=R^{2}+r^{2}-r R \sqrt{3}$ and $1=L^{2}+\ell^{2}+2 \ell L \sqrt{3}$; the latter two are obtained by using the law of cosines in the triangles with vertices $\rho\left(u_{0}\right) \overrightarrow{0} \rho\left(u_{1}\right)$ and $\rho\left(v_{0}\right) \overrightarrow{0} \rho\left(v_{5}\right)$, respectively. This system of equations has essentially a unique solution $R=(6+7 \sqrt{3}+\sqrt{15}) / 12 \approx$ 1.833, $r=(6+7 \sqrt{3}-\sqrt{15}) / 12 \approx 1.188, L=R-1$ and $\ell=r-1$, which gives a unit-distance representation of $I(12,1,5)$, seen on Figure 6 , right. 


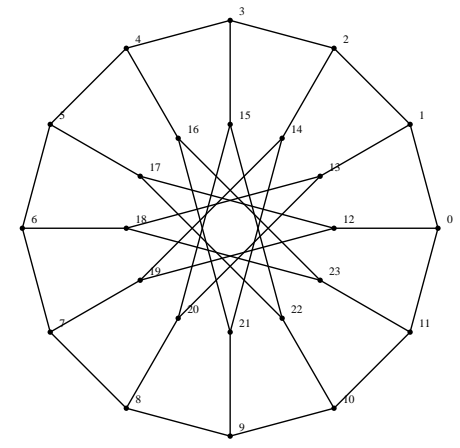

(a)

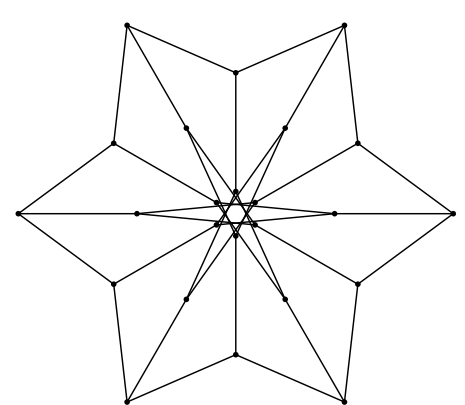

(b)

Figure 6 . The $I$-graph $I(12,1,5)$ (a), drawn with unit distances in the Euclidean plane (b).

Remark 14. It is necessary to find a representation of the $I$-graph $I(12,1,5)$ without using the twist since $I(12,1,5)$ has no admissible isomorph. Namely, the set $\mathbb{Z}_{12}^{*}$ only has four members, $1,5,7$ and 11 . Therefore the only isomorphs $I(n, j, k)$ of $I(12,1,5)$ have $j \in\{1,11\}$ and $k \in\{5,7\}$ or vice versa by Theorem 5 . The radius of the circle determined by the parameters 1 and 11 equals $R=\frac{\sqrt{2}}{-1+\sqrt{3}}$ while the parameters 5 and 7 determine the radius of $r=\frac{\sqrt{2}}{1+\sqrt{3}}$. Therefore for any choice of parameters of isomorphic $I$-graphs we have $|R-r|=$ $\sqrt{2}$. Hence, these graphs are all inadmissible by Theorem 3 .

Proof of Theorem 1. The $I$-graphs $I(n, j, j)$ have a unit-distance representation in the plane that is not vertex-degenerate by Proposition 12 . The $I$ graphs $I(12 m, m, 5 m)$ or $I(12 m, 5 m, m)$, where $m \in \mathbb{N}$, have a unit-distance representation in the plane by Proposition 11 and Proposition 13. For other $I$-graphs the assertion follows by Theorem 5 and Theorem 6 .

\section{Conclusion}

Theorem 1 shows each $I$-graph has a unit-distance representation that is not vertex-degenerate. Since each generalized Petersen graph is an $I$-graph, we obtain the following important consequence.

Corollary 15. Each generalized Petersen graph admits a non-vertex-degenerate unit-distance representation in the plane.

Although this is a positive result, a word of caution is in place. Figure 7 depicts non-vertex-degenerate unit-distance representations of four $I$-graphs. However, they are all vertex-edge-degenerate. The theory that we have developed does not prevent such degeneracies from happening. Observe that vertex-edge-degenerate situations can occur only in two cases: firstly, when a 

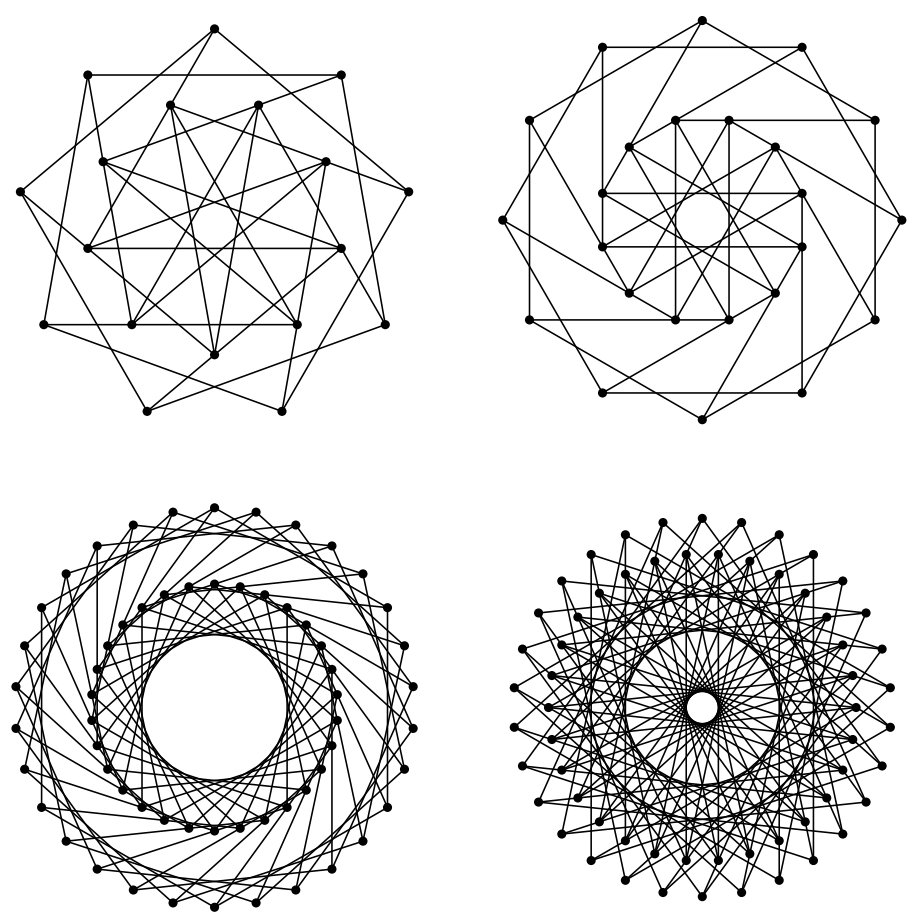

FiguRE 7. Rotational unit-distance representations of $I$ graphs $I(9,2,4), I(12,2,5), I(30,5,9), I(30,9,14)$ are vertexedge-degenerate.

spoke contains a vertex of the inner rim and secondly, when an edge of the outer rim contains a vertex of the inner rim. The following two propositions from [14] provide necessary and sufficient conditions for checking whether a rotational unit-distance representation of an $I$-graph is vertex-edge-degenerate.

Proposition 16. Let $\rho$ be a unit-distance representation with rotational symmetry of an $I$-graph $I(n, j, k)$ with $r, R$ and $\phi$ as in (3) and (5). Suppose $j<k<n / 2$. Then a vertex of the inner rim lies on a spoke that connects the inner and the outer rim if and only if

$$
\frac{n}{\pi} \arcsin \left(\frac{1+r^{2}-R^{2}}{2 r}\right) \in \mathbb{N}
$$

and $\rho$ is vertex-edge-degenerate.

Proposition 17. Let $\rho$ be a unit-distance representation with rotational symmetry of an I-graph $I(n, j, k)$ with $r, R$ and $\phi$ as in (3) and (5). Suppose $j<k<n / 2$. If $\sqrt{4 R^{2}-1} /(2 r)>1$, then a vertex of the inner rim does not 
lie on an edge of the outer rim. Otherwise let $\beta=\arccos \left(\sqrt{4 R^{2}-1} /(2 r)\right)$ and a vertex of the inner rim lies on an edge of the outer rim if and only if there exists an $a \in \mathbb{N}$ such that

$$
\phi=\frac{\pi}{n}(j+2 a) \pm \beta
$$

and $\rho$ is vertex-edge-degenerate.

These propositions were used as a basis of a computer search for all admissible $I$-graphs with a vertex-edge-degenerate unit-distance representation with rotational symmetry up to 500 vertices. Only four distinct connected cases were discovered, see Figure 7. However, all of them have admissible isomorphs whose unit-distance representations are not vertex-edge degenerate. For example, $I(9,2,4)$ is isomorphic to $I(9,1,4)$ which is admissible by Theorem 3 and non-vertex-edge-degenerate by Proposition 16 and Proposition 17. Similarly, $I(12,2,5)$ is isomorphic to $I(12,1,2), I(30,5,9)$ is isomorphic to $I(30,3,5)$ and $I(30,9,14)$ is isomorphic to $I(30,3,8)$.

Conjecture 1. Let $\rho$ be a unit-distance representation with rotational symmetry of an $I$-graph $I(n, j, k)$ where $j \neq k$ and $\operatorname{gcd}(n, j, k)=1$. If $n>30$, then $\rho$ is not vertex-edge-degenerate.

If the edges in Figure 7 are extended into lines, objects resemble astral configurations of Berman [2] and Grünbaum [12] or polycyclic configurations of Boben and Pisanski [3]. Exploring these intriguing relationships between unit-distance representations of graphs and geometric configurations may be a challenging research project.

Acknowledgements. Research was supported in part by grants P1-0294, J16062, L1-7230 and L1-0696 from Ministry of high education, science and technology of the Republic of Slovenia and by ARRS within the EUROCORES Programme EUROGIGA (project GReGAS) of the European Science Foundation.

\section{References}

[1] B. Alspach, The classification of Hamiltonian generalized Petersen graphs, J. Combin. Theory Ser. B 34 (1983), no. 3, 293-312.

[2] L. W. Berman, A characterization of astral $\left(n_{4}\right)$ configurations, Discrete Comput. Geom. 26 (2001), no. 4, 603-612.

[3] M. Boben and T. Pisanski, Polycyclic configurations, European J. Combin. 24 (2003), no. 4, 431-457.

[4] M. Boben, T. Pisanski, and A. Žitnik, I-graphs and the corresponding configurations, J. Combin. Des. 13 (2005), no. 6, 406-424.

[5] I. Z. Bouwer, W. W. Chernoff, B. Monson, and Z. Star, The Foster Census, Charles Babbage Research Centre, 1988.

[6] F. Buckley and F. Harary, On the Euclidean dimension of a wheel, Graphs Combin. 4 (1988), no. 1, 23-30.

[7] K. B. Chilakamarri, The unit-distance graph problem: A brief survey and some new results, Bull. Inst. Combin. Appl. 8 (1993), 39-60. 
[8] H. S. M. Coxeter, Self-dual configurations and regular graphs, Bull. Amer. Math. Soc. 56 (1950), 413-455.

[9] P. Erdős, F. Harary, and W. T. Tutte, On the dimension of a graph, Mathematika 12 (1965), 118-122.

[10] R. Frucht, J. E. Graver, and M. E. Watkins, The groups of the generalized Petersen graphs, Proc. Cambridge Philos. Soc. 70 (1971), 211-218.

[11] S. V. Gervacio and I. B. Jos, The Euclidean dimension of the join of two cycles, Discrete Math. 308 (2008), no. 23, 5722-5726.

[12] B. Grünbaum, Configurations of Points and Lines, Graduate Studies in Mathematics, 103. American Mathematical Society, Providence, RI, 2009.

[13] H. Hadwiger, Ungelöste probleme no. 40, Elem. Math. 16 (1961), 103-104.

[14] B. Horvat, Unit-distance representations of graphs, Ph.D thesis (in Slovene), University of Ljubljana, 2009.

[15] B. Horvat and T. Pisanski, Unit distance representations of the Petersen graph in the plane, Ars Combin., (to appear).

[16] _ Products of unit distance graphs, Discrete Math. 310 (2010), no. 12, 1783-1792.

[17] B. Horvat, T. Pisanski, and A. Žitnik, Isomorphism checking of I-graphs, Graphs Combin., to appear, doi: 10.1007/s00373-011-1086-2.

[18] M. Lovrečič Saražin, A note on the generalized Petersen graphs that are also Cayley graphs, J. Combin. Theory Ser. B 69 (1997), no. 2, 226-229.

[19] H. Maehara and V. Rödl, On the dimension to represent a graph by a unit distance graph, Graphs Combin. 6 (1990), no. 4, 365-367.

[20] R. Nedela and M. Škoviera, Which generalized Petersen graphs are Cayley graphs?, J. Graph Theory 19 (1995), no. 1, 1-11.

[21] T. D. Parsons and T. Pisanski, Vector representations of graphs, Discrete Math. 78 (1989), no. 1-2, 143-154.

[22] M. Petkovšek and H. Zakrajšek, Enumeration of I-graphs: Burnside does it again, Ars Math. Contemp. 2 (2009), no. 2, 241-262.

[23] T. Pisanski and A. Žitnik, Representing Graphs and Maps, Topics in Topological Graph Theory, Series: Encyclopedia of Mathematics and its Applications, No. 129. Cambridge University Press, 2009.

[24] A. Soifer, The Mathematical Coloring Book: Mathematics of Coloring and the Colorful Life of Its Creators, Springer, illustrated edition, 2008.

[25] A. Steimle and W. Staton, The isomorphism classes of the generalized Petersen graphs, Discrete Math. 309 (2009), no. 1, 231-237.

[26] M. E. Watkins, A theorem on Tait colorings with an application to the generalized Petersen graphs, J. Combinatorial Theory 6 (1969), 152-164.

\author{
ARJANA ŽITNIK \\ IMFM \\ UNIVERSITY OF LJUBLJANA \\ SI-1111 Ljubluana, Slovenia \\ E-mail address: Arjana.Zitnik@fmf.uni-lj.si \\ BORIS HORVAT \\ IMFM \\ UNIVERSITY OF LJUBLJANA \\ SI-1111 LJubluana, Slovenia \\ E-mail address: Boris.Horvat@fmf.uni-1j.si
}


TOMAŽ PISANSKI

IMFM

University of LJUbluana AND University of Primorska

SI-1111 LuUbluana, Slovenia

E-mail address: Tomaz.Pisanski@fmf.uni-lj.si 